\title{
Existence of Secondary Bifurcations or Isolas for PDEs
}

\author{
Marcio Gameiro* Jean-Philippe Lessard ${ }^{\dagger}$
}

\begin{abstract}
In this paper, we introduce a method to conclude about the existence of secondary bifurcations or isolas of steady state solutions for parameter dependent nonlinear partial differential equations. The technique combines the Global Bifurcation Theorem, knowledge about non existence of nontrivial steady state solutions at the zero parameter value and explicit information about the coexistence of multiple nontrivial steady states at a positive parameter value. We apply the method to the two-dimensional Swift-Hohenberg equation.
\end{abstract}

\section{Introduction}

Studying the global structure of solution sets of nonlinear eigenvalue problems in a Banach space is a difficult problem. Powerful techniques from global analysis like the Global Bifurcation Theorem of Rabinowitz [1] allow one to conclude about existence of continua of solutions of these equations and provide information about the global behavior of these continua (e.g. unboundedness). However, if one is interested in answering specific questions regarding the structure of the solution sets, then these analytical methods soon become inefficient. In particular, one cannot use the Global Bifurcation Theorem to conclude about the existence of secondary bifurcations or existence of solution sets disjoint from the trivial solution set (isolas). For that reason, numerical simulations are often used to get new insights and observations in order to conjecture the answer to these questions.

In this paper, we propose to merge the strengths of global analysis and rigorous computations to conclude about the existence of secondary bifurcations or isolas of steady states for parameter dependent nonlinear partial differential equations (PDEs) of the form

$$
u_{t}=E(u, \nu), \quad \nu \in \mathbb{R}
$$

More specifically, we first use the so-called validated continuation method for high-dimensional PDEs introduced in [2] to prove coexistence of multiple nontrivial steady state solutions of (1) at a parameter value $\nu^{*}>0$. Using these solutions plus knowledge about non existence of nontrivial steady state solutions at $\nu=0$, one can then use the Global Bifurcation Theorem introduced in [1] to prove existence of isolas or secondary bifurcations.

Even though this method is rather general, we choose to present it in the context of a

${ }^{*}$ Department of Mathematics, Kyoto University, Kyoto, 606-8502, Japan (gameiro@math.kyoto-u.ac.jp).

${ }^{\dagger}$ BCAM - Basque Center for Applied Mathematics, Bizkaia Technology Park, 48160 Derio, Bizkaia, Spain. and Rutgers University, Department of Mathematics, 110 Frelinghuysen Rd, Piscataway, NJ 08854, USA (lessard@math.rutgers.edu). This author was partially supported by NSF grant DMS-0511115, by DARPA, and by DOE grant DE-FG02-05ER25711. 
specific problem. More precisely, consider the Swift-Hohenberg PDE

$$
\begin{aligned}
& u_{t}=\nu u-(1+\Delta)^{2} u-u^{3}, \text { in } \Omega=\left[0, \ell_{1}\right] \times\left[0, \ell_{2}\right], \\
& u(x, y, t)=u\left(x+\ell_{1}, y, t\right)=u\left(x, y+\ell_{2}, t\right), \\
& u(x, y, t)=u(-x, y, t)=u(x,-y, t)=u(-x,-y, t),
\end{aligned}
$$

with periodic boundary conditions on the two dimensional rectangle $\Omega$ restricited to even symmetries. The Swift-Hohenberg PDE was originally introduced as a one-dimensional model in [3] to describe the onset of Rayleigh-Bénard convection and is widely used as model for pattern formation.

Theorem 1.1. At least one of the following two statements is true:

1. Equation (2) undergoes a secondary bifurcation of steady states;

2. Equation (2) possesses an isola, that is, a connected set of steady states disjoint from the trivial solution set $\mathcal{E}_{0} \stackrel{\text { def }}{=}\{(0, \nu) \mid \nu \in \mathbb{R}\}$.

The proof of Theorem 1.1 requires a combination of the Global Bifurcation Theorem, global knowledge about non existence of nontrivial steady state solutions at the parameter value $\nu=0$ and coexistence of multiple nontrivial steady states at a parameter value $\nu^{*}>0$.

The paper is organized as follows. In Section 2, we recall the Global Bifurcation Theorem of Rabinowitz [1] and apply it to the two-dimensional Swift-Hohenberg equation (2). In Section 3, we use the energy functional associated to (2) to prove that the Swift-Hohenberg equation has no nontrivial steady states solutions at the parameter value $\nu=0$. In Section 4 , we use the so-called validated continuation for high dimensional PDEs method developed in [2] to prove existence of six distinct steady states for a parameter value $\nu^{*}>0$. Finally, combining all the above mentioned ingredients, we prove Theorem 1.1 in Section 5.

\section{The Global Bifurcation Theorem}

The following result, due to Rabinowitz, is introduced and proved in [1].

Theorem 2.1 (Global Bifurcation). Suppose that $L$ is a compact operator on a Banach space $X$, and $H(u, \lambda)$ is a compact operator on $X \times \mathbb{R}$. If $\lambda_{0}$ is a characteristic value of $L$ with odd algebraic multiplicity, then $\left(0, \lambda_{0}\right)$ is a bifurcation point of

$$
F(u, \lambda) \stackrel{\text { def }}{=} u-\lambda L u-H(u, \lambda)=0 .
$$

Moreover, if $\Sigma$ is the set of the nontrivial solutions of $F(u, \lambda)=0$, then there is a closed connected component $\Sigma_{1}$ of $\bar{\Sigma}$, such that $\left(0, \lambda_{0}\right) \in \Sigma_{1}$, and either (a) $\Sigma_{1}$ is unbounded; or (b) $\Sigma_{1}$ contains $\left(0, \lambda_{*}\right)$, where $\lambda_{*} \neq \lambda_{0}$ is also a characteristic value of $L$.

We want to apply Theorem 2.1 to the Swift-Hohenberg equation (2). First we define the linear operator $L$. For that purpose, fix $\varepsilon \in(0,1]$ and define

$$
A u \stackrel{\text { def }}{=}\left[(1+\Delta)^{2}+\varepsilon\right] u
$$

Since the Swift-Hohenberg equation is considered with periodic boundary conditions on the rectangle $\Omega=\left[0, \ell_{1}\right] \times\left[0, \ell_{2}\right]$, we identify the domain with the two-dimensional torus $\mathbb{T}^{2}$.

Letting $L_{1}=\frac{2 \pi}{\ell_{1}}$ and $L_{2}=\frac{2 \pi}{\ell_{2}}$, one denotes $\mathbb{T}^{2}=\left[0, \frac{2 \pi}{L_{1}}\right] \times\left[0, \frac{2 \pi}{L_{2}}\right]$. For a function $u$ defined 
on $\mathbb{T}^{2}$, we use the notation $\hat{u}(\boldsymbol{k})$ to denote the Fourier coefficient associated to the mode $\boldsymbol{k}=\left(k_{1}, k_{2}\right)$ with basis element $e^{i k_{1} L_{1} x} e^{i k_{2} L_{2} y}$. Consider then $H^{s}\left(\mathbb{T}^{2}\right)=W^{s, 2}\left(\mathbb{T}^{2}\right) \subset L^{2}\left(\mathbb{T}^{2}\right)$ the Sobolev space of functions defined on $\mathbb{T}^{2}$ with norm

$$
\|u\|_{H^{s}\left(\mathbb{T}^{2}\right)}^{2}=\sum_{\boldsymbol{k} \in \mathbb{Z}^{2}}\left(1+\|\boldsymbol{k}\|^{2}\right)^{s}|\hat{u}(\boldsymbol{k})|^{2},
$$

where $\|\boldsymbol{k}\|=\sqrt{k_{1}^{2}+k_{2}^{2}}$.

Lemma 2.2. The linear operator $A$ defined by (6) maps $H^{5}\left(\mathbb{T}^{2}\right)$ into $H^{1}\left(\mathbb{T}^{2}\right)$ and it is invertible. Denote by $A^{-1}$ its inverse. Then $A^{-1}$ maps $H^{1}\left(\mathbb{T}^{2}\right)$ into $H^{5}\left(\mathbb{T}^{2}\right)$.

Proof. Denote $\boldsymbol{k}=\left(k_{1}, k_{2}\right) \in \mathbb{Z}^{2}$ and $\boldsymbol{k} \boldsymbol{L}=\left(k_{1} L_{1}, k_{2} L_{2}\right)$. Recalling (6), one has that

$$
\widehat{A u}(\boldsymbol{k})=\left(\left(1-\|\boldsymbol{k} \boldsymbol{L}\|^{2}\right)^{2}+\varepsilon\right) \hat{u}(\boldsymbol{k}) .
$$

Consider $u \in H^{5}\left(\mathbb{T}^{2}\right)$ and define $L_{*}=\max \left\{\left|L_{1}\right|,\left|L_{2}\right|\right\}$. Since $\varepsilon \in(0,1]$, one has that

$$
\begin{aligned}
\|A u\|_{H^{1}\left(\mathbb{T}^{2}\right)}^{2} & =\sum_{\boldsymbol{k} \in \mathbb{Z}^{2}}\left(1+\|\boldsymbol{k}\|^{2}\right)|\widehat{A u}(\boldsymbol{k})|^{2} \\
& =\sum_{\boldsymbol{k} \in \mathbb{Z}^{2}}\left(1+\|\boldsymbol{k}\|^{2}\right)\left(\left(1-\|\boldsymbol{k} \boldsymbol{L}\|^{2}\right)^{2}+\varepsilon\right)^{2}|\hat{u}(\boldsymbol{k})|^{2} \\
& \leq 2 \sum_{\boldsymbol{k} \in \mathbb{Z}^{2}}\left(1+\|\boldsymbol{k}\|^{2}\right)\left(1+\|\boldsymbol{k} \boldsymbol{L}\|^{2}\right)^{4}|\hat{u}(\boldsymbol{k})|^{2} \\
& \leq 2 \gamma\|u\|_{H^{5}\left(\mathbb{T}^{2}\right)}^{2}<\infty,
\end{aligned}
$$

where $\gamma=\gamma\left(L_{*}\right)$ is a positive constant depending on $L_{*}$. Hence, $A$ maps $H^{5}\left(\mathbb{T}^{2}\right)$ into $H^{1}\left(\mathbb{T}^{2}\right)$. Let us now show that $A$ is invertible. We want to show that the problem

$$
A u=v
$$

has a unique solution $u$ defined in $\mathbb{T}^{2}$. Computing the Fourier expansion on both sides of (7) gives

$$
\widehat{A u}(\boldsymbol{k})=\left[\left(1-\|\boldsymbol{k} \boldsymbol{L}\|^{2}\right)^{2}+\varepsilon\right] \hat{u}(\boldsymbol{k})=\hat{v}(\boldsymbol{k}) .
$$

Since $\left(1-\|\boldsymbol{k} \boldsymbol{L}\|^{2}\right)^{2}+\varepsilon>0$, for all $\boldsymbol{k} \in \mathbb{Z}^{2}$, one can uniquely define the solution $u$ of (7) by

$$
u=\sum_{\boldsymbol{k} \in \mathbb{Z}^{2}} \hat{u}(\boldsymbol{k}) \psi_{\boldsymbol{k}}, \quad \text { with } \hat{u}(\boldsymbol{k})=\frac{\hat{v}(\boldsymbol{k})}{\left(1-\|\boldsymbol{k} \boldsymbol{L}\|^{2}\right)^{2}+\varepsilon},
$$

where $\psi_{\boldsymbol{k}}=\psi_{k_{1}, k_{2}}=e^{i k_{1} L_{1} x} e^{i k_{2} L_{2} y}$. Hence, the operator $A$ is invertible. Denote by $A^{-1}$ its inverse. The fact that $A^{-1}$ maps $H^{1}\left(\mathbb{T}^{2}\right)$ into $H^{5}\left(\mathbb{T}^{2}\right)$ follows by similar arguments to the one used above.

Let us now put the Swift-Hohenberg equation (2) in the context of Theorem 2.1. First, one needs to define the compact operators $L$ and $H$. By the Sobolev Embedding Theorem, there exists a compact inclusion $i_{1}: H^{5}\left(\mathbb{T}^{2}\right) \rightarrow H^{1}\left(\mathbb{T}^{2}\right)$. Defining $L \stackrel{\text { def }}{=} i_{1} \circ A^{-1}$ and $X \stackrel{\text { def }}{=} H^{1}\left(\mathbb{T}^{2}\right)$, one has that the linear operator $L: X \rightarrow X$ is compact. Now, let us construct $H: X \rightarrow X$. For $u \in X=H^{1}\left(\mathbb{T}^{2}\right)$, one has that $u \in L^{6}\left(\mathbb{T}^{2}\right)$, which then implies that $u^{3} \in L^{2}\left(\mathbb{T}^{2}\right)$. Now, a solution $w$ of $A w=u^{3}$ is such that $w \in H^{4}\left(\mathbb{T}^{2}\right)$. This can be 
proved by computing the Fourier expansion like in the proof of Lemma 2.2. Hence, one has that $A^{-1} u^{3} \in H^{4}\left(\mathbb{T}^{2}\right)$. By the Sobolev Embedding Theorem, consider the compact inclusion $i_{2}: H^{4}\left(\mathbb{T}^{2}\right) \rightarrow H^{1}\left(\mathbb{T}^{2}\right)$. Defining $H(u) \stackrel{\text { def }}{=} i_{2} \circ A^{-1} u^{3}$, one has that $H: X \rightarrow X$ is compact.

Let us now define $\lambda \stackrel{\text { def }}{=} \nu+\varepsilon$. Hence, solving for the steady states of (2) is equivalent to solve for

$$
F(u, \lambda) \stackrel{\text { def }}{=} u-\lambda L u-H(u)=0
$$

One can then apply Theorem 2.1 to the Swift-Hohenberg equation (2) defined on the twodimensional torus.

Corollary 2.3. If $\lambda_{0}$ is a characteristic value of $L=i_{1} \circ A^{-1}$ with odd algebraic multiplicity, then $\left(0, \lambda_{0}\right)$ is a bifurcating steady state of (2). Moreover, if $\Sigma$ is the set of the nontrivial steady states of (2), then there is a closed connected component $\Sigma_{1}$ of $\bar{\Sigma}$, such that $\left(0, \lambda_{0}\right) \in$ $\Sigma_{1}$, and either (a) $\Sigma_{1}$ is unbounded; or (b) $\Sigma_{1}$ contains $\left(0, \lambda_{*}\right)$, where $\lambda_{*} \neq \lambda_{0}$ is also a characteristic value of $L$.

\section{Global uniqueness of steady state at $\nu=0$}

In this Section, we prove that there are no nontrivial steady state solutions at the parameter value $\nu=0$.

Lemma 3.1. The trivial solution $u=0$ is the unique steady state solution of the SwiftHohenberg PDE (2) at the parameter value $\nu=0$.

Proof. First, it is well known that the energy functional

$$
E(u) \stackrel{\text { def }}{=} \iint_{\mathbb{T}^{2}}\left[\frac{1}{4} u^{4}+\frac{1}{2}((1+\Delta) u)^{2}\right] d A
$$

acts as a Lyapunov function for the Swift-Hohenberg equation (2) at $\nu=0$ subject to the symmetries (4) (see e.g. [4]). Hence, steady state solutions of (2) corresponds to minimizers of the action functional (8). Assume now that there exists a local minimizer $\bar{u} \neq 0$. Then, there is a neighborhood $N$ of $\bar{u}$ in $X$ such that $E(\bar{u}) \leq E(v)$ for all $v \in N$. Considering a general $u \neq \bar{u} \in X$, there exists $\delta \in(0,1)$ such that $u_{\delta} \stackrel{\text { def }}{=}(1-\delta) \bar{u}+\delta u \in N$. Since the functional (8) is strictly convex, that implies that $E(\bar{u}) \leq E\left(u_{\delta}\right)<(1-\delta) E(\bar{u})+\delta E(u)$. Hence, $\delta E(\bar{u})<\delta E(u)$. In particular, for $u=0$, one get that $0 \leq E(\bar{u})<E(0)=0$. This is a contradiction. Hence, $u=0$ is the unique minimizer. That shows that the trivial solution $u=0$ is the unique steady state solution at $\nu=0$.

\section{Coexistence of multiple steady states at $\nu^{*}>0$}

In this Section, we use the so-called validated continuation technique to demonstrate the coexistence of six steady state solutions of (2) at the parameter value $\nu^{*}=0.8$. Validated continuation $[5,6,7,2,8,9,10]$ is a computational method to rigorously compute solutions of infinite dimensional parameter dependent nonlinear operators of the form $f(a, \nu)=0$. The idea of the method is to use $f$ to construct a Newton-like operator $T$ in a Banach space, and then to combine analytic a priori estimates and rigorous computation to show that $T$ is a contraction mapping. It is important to note that since the validated continuation technique is based on the Implicit Function Theorem, it cannot be used to handle bifurcations directly. In this paper, we use the work presented in [2], which was introduced to rigorously compute 
steady state solutions of high-dimensional PDEs. To apply this computational method, one first needs to set up the operator $f$.

Notice first that due to the boundary conditions (3) and the symmetry assumptions (4), one can expand the steady state solutions of (2) using a cosine basis $\left\{\psi_{\boldsymbol{k}}\right\}_{\boldsymbol{k} \in \mathbb{N}^{2}}$ given by

$$
\psi_{\boldsymbol{k}}(y) \stackrel{\text { def }}{=} \cos \left(k_{1} L_{1} x\right) \cos \left(k_{2} L_{2} y\right)
$$

Hence, using the expansion

$$
u=\sum_{\boldsymbol{k} \in \mathbb{Z}^{2}} a_{\boldsymbol{k}} \psi_{\boldsymbol{k}}
$$

with the assumption that $a_{|\boldsymbol{k}|}=a_{\boldsymbol{k}}$ for $\boldsymbol{k} \in \mathbb{Z}^{2}$, the expansion of the steady states of (2) takes the form

$$
f_{\boldsymbol{k}} \stackrel{\text { def }}{=} \mu_{\boldsymbol{k}} a_{\boldsymbol{k}}-\sum_{\substack{\boldsymbol{k}^{1}+\boldsymbol{k}^{2}+\boldsymbol{k}^{3}=\boldsymbol{k} \\ \boldsymbol{k}^{j} \in \mathbb{Z}^{d}}} a_{\boldsymbol{k}^{1}} a_{\boldsymbol{k}^{2}} a_{\boldsymbol{k}^{3}},
$$

where

$$
\mu_{\boldsymbol{k}}(\nu) \stackrel{\text { def }}{=} \nu-\left[1-\left(k_{1}^{2} L_{1}^{2}+k_{2}^{2} L_{2}^{2}\right)\right]^{2},
$$

with $f_{|\boldsymbol{k}|}=f_{\boldsymbol{k}}$, for all $\boldsymbol{k} \in \mathbb{Z}^{2}$. Therefore, we only need to solve $f_{\boldsymbol{k}}=0$ for $\boldsymbol{k} \geq \mathbf{0}$. Hence, defining $a=\left\{a_{\boldsymbol{k}}\right\}_{\boldsymbol{k} \in \mathbb{N}^{2}}$ and $f=\left\{f_{\boldsymbol{k}}\right\}_{\boldsymbol{k} \in \mathbb{N}^{2}}$, it can be shown that finding steady states of (2) is equivalent to finding solutions of

$$
f(a, \nu)=0
$$

in a space of algebraically decaying coefficients (see [2]). We have now the following result.

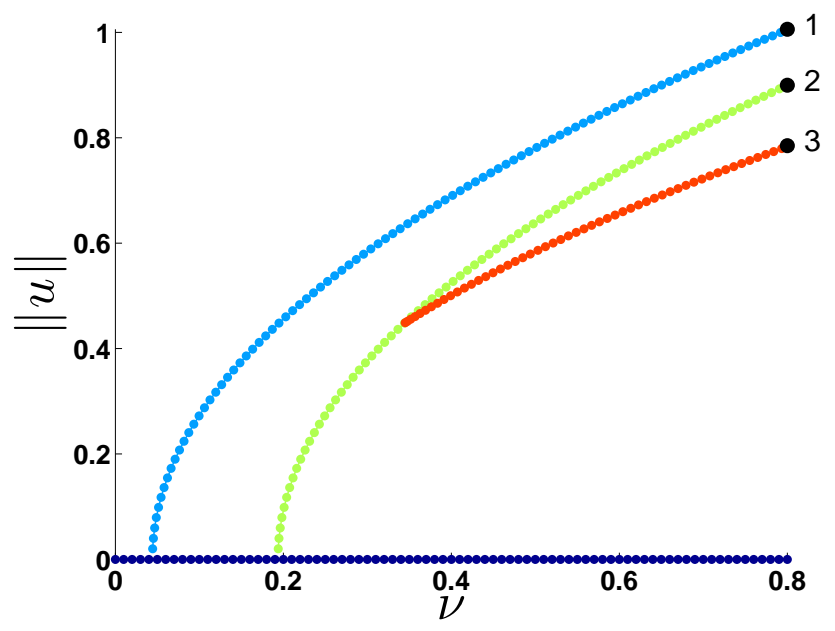

Figure 1: Diagram of rigorously computed steady states of (2) with $\ell_{1}=\frac{2 \pi}{1.1}$ and $\ell_{2}=\frac{2 \pi}{1.2}$ for some values of $\nu \in[0,0.8]$.

Theorem 4.1. Consider the Swift-Hohenberg equation (2) with $\ell_{1}=\frac{2 \pi}{1.1}$ and $\ell_{2}=\frac{2 \pi}{1.2}$. At the parameter value $\nu^{*}=0.8$, equation (2) has at least six nontrivial distinct steady state solutions. 
Proof. For $\nu^{*}=0.8$, the rigorous computational method from [2] was used to prove existence and local uniqueness of three solutions of (10), corresponding to steady state solutions of (2). For each one of these solutions there is the corresponding solution given by the reflection symmetry $(u \mapsto-u)$. Rigorous computations were used to verify that these six steady state solutions are distinct.

Note that we actually proved more than the result of Theorem 4.1. In fact, in order to get to the parameter value $\nu^{*}=0.8$, we proved the existence of many more steady state solutions. In Figure 1, we show the diagram of all rigorously computed solutions. However, as shown in Section 5, the proof of Theorem 1.1 only requires knowledge of coexistence at $\nu^{*}=0.8$. As mentioned before, since the validate continuation technique is based on the Implicit Function Theorem, it can not be used to handle bifurcations. Hence all the rigorously computed solutions presented in Figure 1 are away from bifurcations.
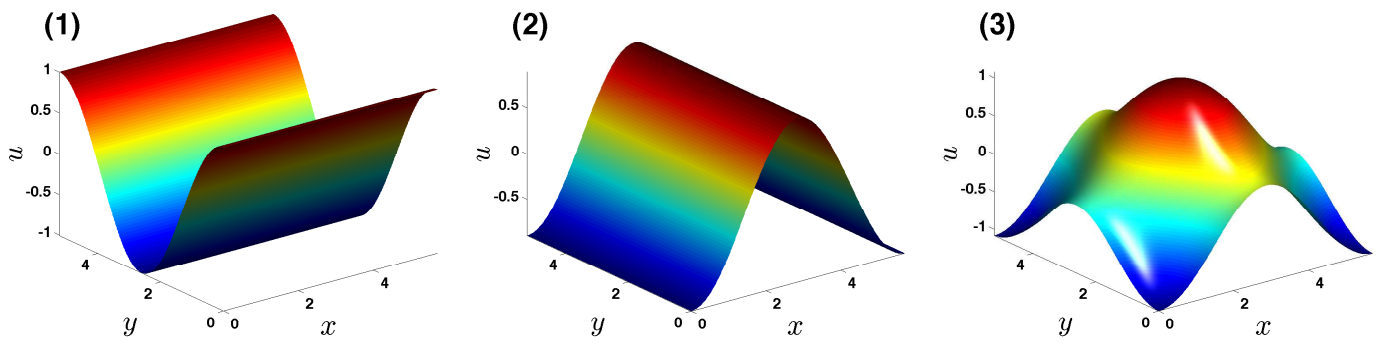

Figure 2: Plot of the three computed nontrivial steady states solutions of the SwiftHohneberg equation (2) defined on the rectangle $\left[0, \frac{2 \pi}{1.1}\right] \times\left[0, \frac{2 \pi}{1.2}\right]$ at the parameter value $\nu^{*}=0.8$.

\section{Proof of the main result}

Proof of Theorem 1.1. Let $\nu^{*}=0.8$, and assume that the theorem is false. Notice that the bifurcations from the trivial solution occur for the values of $\nu$ given by

$$
\nu_{k_{1}, k_{2}}=\left[1-\left(k_{1}^{2} L_{1}^{2}+k_{2}^{2} L_{2}^{2}\right)\right]^{2} .
$$

It is easy to see that in the parameter interval $\left[0, \nu^{*}\right]$, the only bifurcations from the trivial solution occur at $\nu_{1}=(0.21)^{2} \approx 0.0441$ and $\nu_{2}=(0.44)^{2} \approx 0.1936$. These bifurcations correspond to the modes $\left(k_{1}, k_{2}\right)=(1,0)$ and $\left(k_{1}, k_{2}\right)=(0,1)$ respectively. Hence, by the reflection symmetry $u \mapsto-u$ on the solutions of (2), there are exactly four branches of equilibria bifurcating from the trivial solution in the interval $\left[0, \nu^{*}\right]$. Denote these branches by $\Sigma_{1}^{ \pm}$and $\Sigma_{2}^{ \pm}$.

In Theorem 4.1 it was proved that there are at least six nontrivial distinct steady state solutions at $\nu^{*}=0.8$. Therefore there is a nontrivial steady state solution $u_{3}$ that does not belong to $\Sigma_{1}^{ \pm} \cup \Sigma_{2}^{ \pm}$. Let $\mathcal{E} \stackrel{\text { def }}{=}\{(u, \nu) \mid F(u, \nu)=0, \nu \in \mathbb{R}\}$ be the set of steady state solutions of (2), and let $\mathcal{E}_{3} \subset \mathcal{E}$ be the connected component that contains $u_{3}$.

Since by assumption there are no isolas and no secondary bifurcations, there must exist a branch of solutions $\Sigma_{3} \subset \mathcal{E}_{3}$ such that $u_{3} \in \Sigma_{3}$, and $\Sigma_{3}$ is connected to the set of trivial solutions $\mathcal{E}_{0}$ at some value of $\nu_{3} \notin\left[0, \nu^{*}\right]$, that is, $\left(\nu_{3}, 0\right) \in \Sigma_{3}$. Then, by Corollary 2.3 , we 
have that either $\Sigma_{3}$ is unbounded, or there exists $\nu_{4} \neq \nu_{3}$ such that $\left(\nu_{4}, 0\right) \in \Sigma_{3}$. Since, by Lemma 3.1, at $\nu_{0}=0$ the only steady state solution is the trivial solution we must have that $\nu_{3}>\nu^{*}$ and $\nu_{4}>\nu^{*}$. In either case, since $\Sigma_{3}$ can not cross the axis $\nu=0$, it must undergo a secondary saddle-node like bifurcation, which contradicts the initial assumption.

\section{Acknowledgments}

We would like to thank Konstantin Mischaikow and Jan Bouwe van den Berg for helpful discussions.

\section{References}

[1] Paul H. Rabinowitz. Some global results for nonlinear eigenvalue problems. J. Functional Analysis, 7:487-513, 1971.

[2] M. Gameiro and J.-P. Lessard. A priori estimates and validated continuation for equilibria of high dimensional PDEs. Submitted, 2009.

[3] J.B. Swift and P.C. Hohenberg. Hydrodynamic fluctuations at the convective instability. Phys. Rev. A, 15(1), 1977.

[4] C. I. Christov and J. Pontes. Numerical scheme for Swift-Hohenberg equation with strict implementation of Lyapunov functional. Math. Comput. Modelling, 35(1-2):8799, 2002.

[5] J.B. van den Berg and J.-P. Lessard. Chaotic braided solutions via rigorous numerics: chaos in the Swift-Hohenberg equation. SIAM J. Appl. Dyn. Syst., 7(3):988-1031, 2008.

[6] J.B. van den Berg, J.-P. Lessard, and K. Mischaikow. Global smooth solution curves using rigorous branch following. Math. Comp., 2009.

[7] S. Day, J.-P. Lessard, and K. Mischaikow. Validated continuation for equilibria of PDEs. SIAM J. Numer. Anal., 45(4):1398-1424 (electronic), 2007.

[8] M. Gameiro and J.-P. Lessard. Rigorous computation of smooth branches of equilibria for the three-dimensional Cahn-Hilliard equation. Submitted, 2009.

[9] M. Gameiro, J.-P. Lessard, and K. Mischaikow. Validated continuation over large parameter ranges for equilibria of PDEs. Math. Comput. Simulation, 79(4):1368-1382, 2008.

[10] J.-P. Lessard. Recent advances about the uniqueness of the slowly oscillating periodic solutions of Wright's equation. J. Differential Equations, 2009. 